\title{
Endovascular Management of Iatrogenic Internal Jugular Vein-Subclavian Artery Fistula Formed by Inadvertently Misplaced Hemodialysis Catheter: A Case Report
}

\author{
Mohammad Gharib Salehi ${ }^{1,{ }^{*}}$, Alireza Rai $^{2}$, Mohammadreza Sobhiyeh $^{3}$ and Elham Shobeiri ${ }^{1}$ \\ ${ }^{1}$ Department of Radiology, Imam Reza Hospital, Kermanshah University of Medical Sciences, Kermanshah, Iran \\ ${ }^{2}$ Department of Cardiology, Imam Ali Hospital, Kermanshah University of Medical Sciences, Kermanshah, Iran \\ ${ }^{3}$ Department of Surgery, Imam Ali Hospital, Kermanshah University of Medical Sciences, Kermanshah, Iran \\ "Corresponding author: Mohammad Gharib Salehi, Department of Radiology, Imam Reza Hospital, Kermanshah University of Medical Sciences, Kermanshah, Iran. Tel: \\ +98-9183750503, E-mail: drmsale2000@yahoo.com
}

Received 2017 July 10; Revised 2018 April 26; Accepted 2018 May 06.

\begin{abstract}
Right internal jugular vein catheterization is a way to establish a temporary dialysis vascular access. Arterial injuries including inadvertent arterial cannulation and/or arteriovenous fistula formation are one of the most clinically important complications of hemodialysis catheter placement. Endovascular management of these conditions is a promising treatment option, especially for aged and co-morbid patients. This report represents a case of internal jugular vein-subclavian artery fistula formed by inadvertently misplaced double lumen tunneled cuffed central venous catheter that was treated successfully with covered stent graft placement.
\end{abstract}

Keywords: Arteriovenous Fistula, Central Venous Catheterization, Subclavian Artery, Endovascular Procedures

\section{Introduction}

Central venous catheterization is a common clinical procedure to establish a temporary dialysis vascular access. Right internal jugular (RIJ) vein is the preferred site of hemodialysis catheter placement (1). Various complications are associated with RIJ catheterization; of particular significance are arterial injuries (e.g. massive bleeding, inadvertent arterial cannulation, arteriovenous fistula formation, and pseudoaneurysm) $(2,3)$. The incidence of serious arterial trauma after RIJ cannulation is seldom (about1/1800) and subclavian artery injuries are even more rare; these complications may be fatal (3). Endovascular treatment has a promising role in the management of such conditions $(2,4)$. We report endovascular management of a case of internal jugular vein-subclavian artery fistula formed by inadvertently misplaced double lumen tunneled cuffed central venous catheter.

\section{Case Presentation}

A female patient was referred to our interventional radiology department for management of inadvertent arterial catheterization after RIJ cannulation. The patient was a 57-year-old white woman with a history of type II diabetes, hypertension, and end stage renal disease (ESRD). She was on hemodialysis three times per week through the formerly placed right jugular vein catheter which was malfunctioned. Earlier that day, the vascular surgeon placed the tunneled RIJ hemodialysis catheter under general anesthesia using ultrasonography guidance and removed the antecedent catheter in the operating room. This procedure was done in a scheduled nonemergency situation. Pulsatile bright red blood backflow through the newly placed catheter was noted in the recovery room. Our team was consulted for removal of the misplaced catheter.

The patient had stable vital signs and was conscious at arrival. Physical examination was unremarkable unless for a bruit over the supraclavicular region, multiple scars and other cutaneous injuries on the right side of the neck due to previous cannulation attempts. Distal pulses were intact; the body mass index (BMI) was 29 and the neck anatomy appeared normal. 12-Fr tunneled double lumen hemodialysis catheter (Arrow; Teleflex, Pennsylvania, United States) was placed through the right internal jugular approach. The patient had one episode of significant bleeding from the puncture site in the recovery unit which dropped her blood pressure and required fluid resuscita- 
tion and direct pressure in order to control the hemorrhage. Post catheterization chest $\mathrm{x}$-ray revealed that the catheter tip was not in the venous system; no other significant finding was noted (e.g. pleural and/or pericardial effusion, and pneumothorax).

CT angiography was performed after suspected inadvertent catheter placement. The catheter entered the subclavian artery slightly distal to the brachiocephalic bifurcation and proximal to the right vertebral artery origin (Figure $1 \mathrm{~A})$.

After administrating 450 milligrams of clopidogrel orally, the patient underwent conventional angiography through femoral artery access under regional anesthesia in order to remark possible percutaneous/endovascular management of the lesion. CT angiography findings were confirmed and the carotid-vertebral systems were normal (Figure 1B). We obtained the written informed consent from the patient and his family concerning potential vertebrobasilar circulation stroke and explained other probable complications including myocardial infarction, excessive uncontrollable hemorrhage, and possible open surgery. The vascular surgical team and their operating theatre were prepared for emergency situations.

First, we decided to attempt both temporary balloon occlusion and external compression simultaneously to obtain hemostasis. Removing the catheter and applying pressure without any other intervention was not an option since there was no direct pressure route and the arterial injury was remarkable. We inflated a $6 \times 60 \mathrm{~mm}$ PTA balloon catheter (FoxCross; Abbott Vascular, Illinois, United States) over the dialysis catheter entry area. At the same time, we withdrew the dialysis catheter and applied external compression on the affected site for 20 minutes under fluoroscopic guidance. This procedure failed to seal the injury and the subsequent angiogram revealed the $4 \mathrm{~mm}$ right subclavian artery defect $13 \mathrm{~mm}$ distal to the brachiocephalic bifurcation and $9 \mathrm{~mm}$ proximal to the right vertebral artery origin. This defect was in connection with the jugular vein and consistent with high flow fistula formation (Figure 2).

Right side subclavian artery branched off the vertebral artery $22 \mathrm{~mm}$ from its origin. Since the left vertebral pathway was the dominant route for posterior circulation and it had no obvious arterial disease on antecedent angiographic examination, in this case, it was not necessary to perform the right vertebral artery occlusion tolerance test prior to sacrificing.

The uppermost arterial diameter at the site of the right subclavian arterial defect was $7 \mathrm{~mm}$ at angiography image. Given that, after intravenous administration of 5000 units of heparin, we introduced $7 \times 37 \mathrm{~mm}$ balloonexpandable covered peripheral stent graft (BeGraft; Bent- ley, Hechinger, Germany) transfemorally and inflated it over the arterial laceration. Having in mind the need for exact stent placement, controlled deployment, and flexible sizing, we preferred balloon-expandable covered stent over the self-expandable stent. Also, using balloon expansion helps to further expand the stent by using larger balloons. The proximal end of the stent was at the right subclavian artery origin and $37 \mathrm{~mm}$ away from the origin was the distal end. The placed stent covered both the arteriovenous fistula and vertebral artery. This intervention also failed. The angiogram revealed continuous extravasation and fistulous communication despite placement of the stent graft, possibly due to both small stent diameter and short stent length selection. The placed stent did not migrate distally (Figure 3 ). So, we chose a wider and longer stent and the second $8 \times 60 \mathrm{~mm}$ balloon expandable covered peripheral stent (Atrium Advanta V12; Maquet Getinge Group, Rastatt, Germany) was placed over the same area again. The proximal end of the stent was at the right subclavian artery origin and $60 \mathrm{~mm}$ away from the origin was the distal end. Finally, total occlusion of the fistula, as well as patency of the right subclavian artery was achieved (Figure 4).

After successful treatment, we started clopidogrel for the patient along with continuing her other medication including lifelong aspirin. The patient was discharged from the hospital after 4 days without any endovascular treatment-related complication. The patient was appointed to regular visits to evaluate new arterial insufficiency symptoms and physical examination of his right hand. Clopidogrel was discontinued after two months. Imaging follow-up after 12 months revealed no significant complications (kinking, fracture, displacement, and significant stenosis) on CT angiogram.

\section{Discussion}

The incidence of serious arterial trauma after RIJ cannulation is seldom (about 1/1800) and subclavian artery injuries are even more rare; these complications (pseudoaneurysm formation, vessel rupture, and frank bleeding) may be fatal (3). There are both surgical and radiointerventional treating options for iatrogenic subclavian artery puncture after venous cannulation with a large bore catheter (5). Although surgical management is the treatment option, with recent technical and device advancements, endovascular procedures are the preferred treatment for RIJ catheterization-related complications. Endovascular treatment has less mortality, and is less invasive $(2,4,5)$. These procedures have been shown to have a good long-term outcome especially for vascular injuries below the clavicle $(3,6,7)$. Infraclavicular vascular injuries 

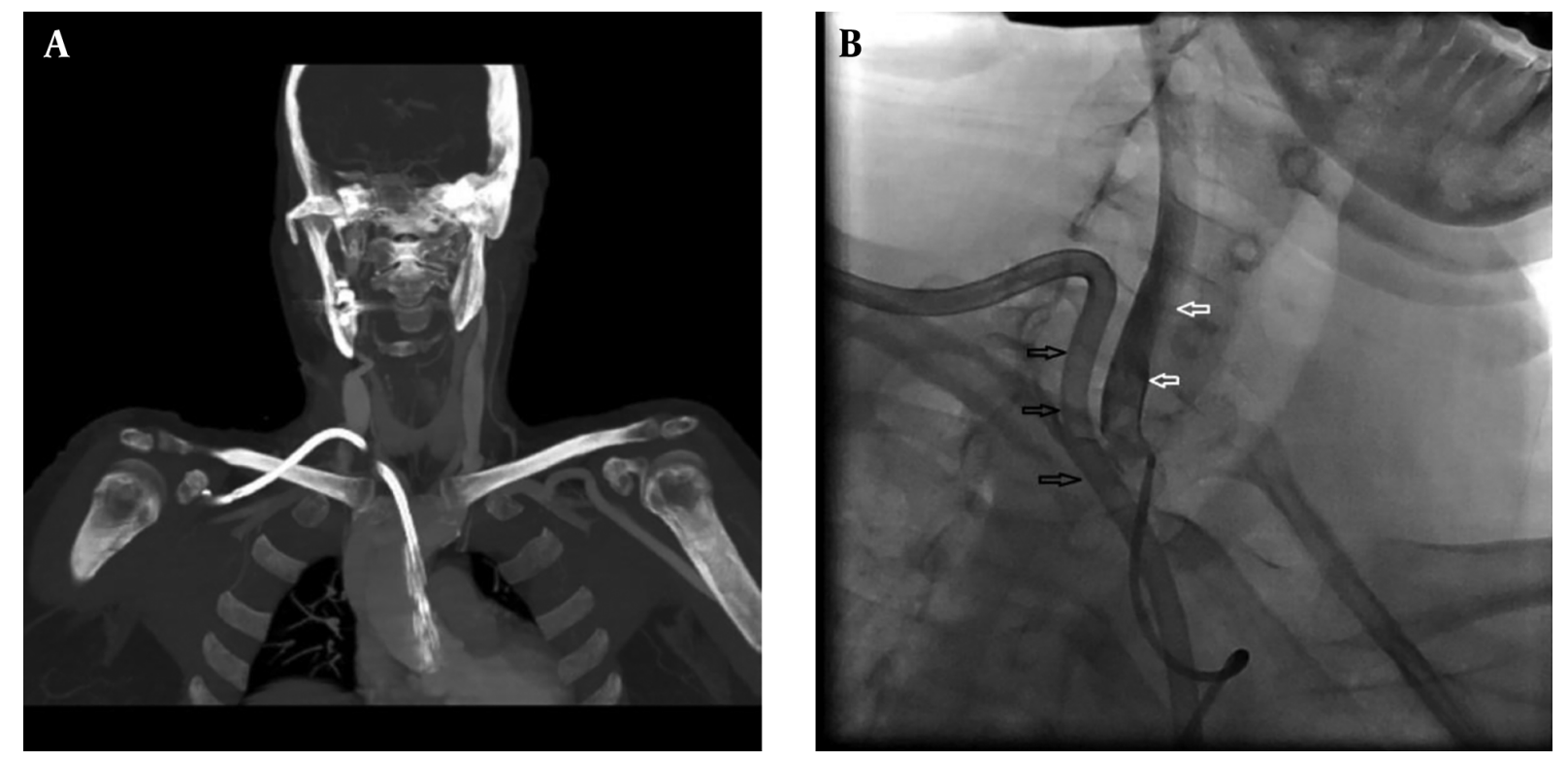

Figure 1. CT angiogram (CTA) of a 57-year-old female patient with significant bleeding after hemodialysis catheter placement showing the catheter (black arrows) penetrating in both internal jugular vein and subclavian artery (white arrows). A, The tip of the catheter is in the ascending aorta over the aortic valve. B, Angiogram confirms CTA findings.

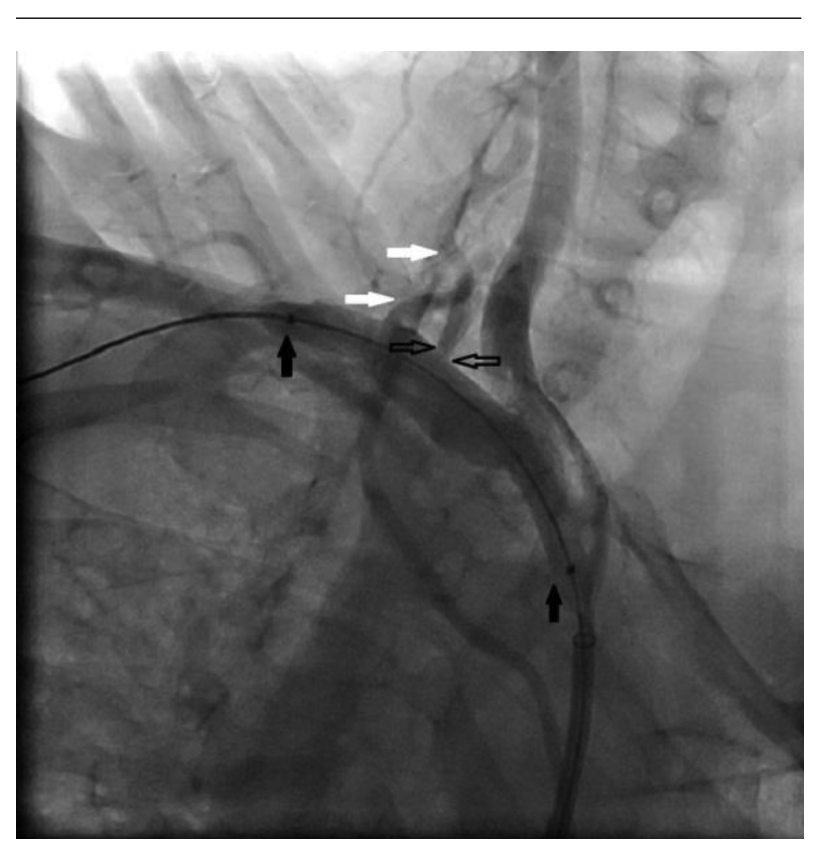

Figure 2. Angiogram after hemodialysis catheter removal showing the balloon occlusion catheter is in place (black arrows), but the arteriovenous communication is still ongoing (open black arrows). Right vertebral artery (white arrows) is also noted.

are hard to approach for vascular surgeons and have more complications.

A variety of applications of various devices such as covered stents, vascular closure devices, tract emboliza-

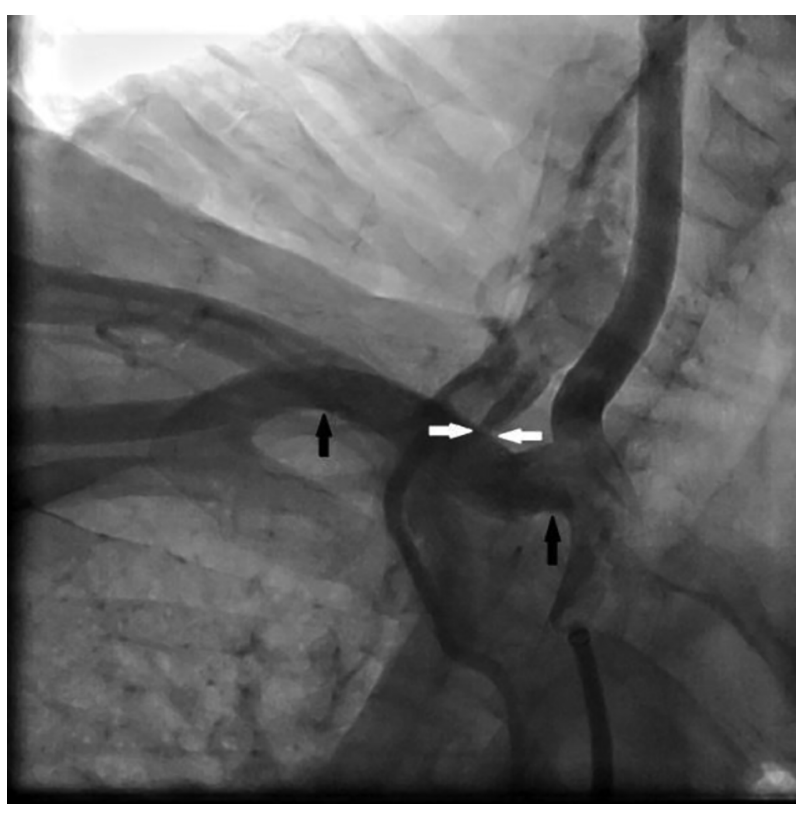

Figure 3. First stent has landed in its proper site (black arrows) but the arteriovenous connection is not sealed (white arrows)

tion, and balloon tamponade have been described to treat complications related to inadvertent subclavian arterial injuries that arise during central venous catheter placement $(2,4,5)$. Each device or treatment option is tailored 


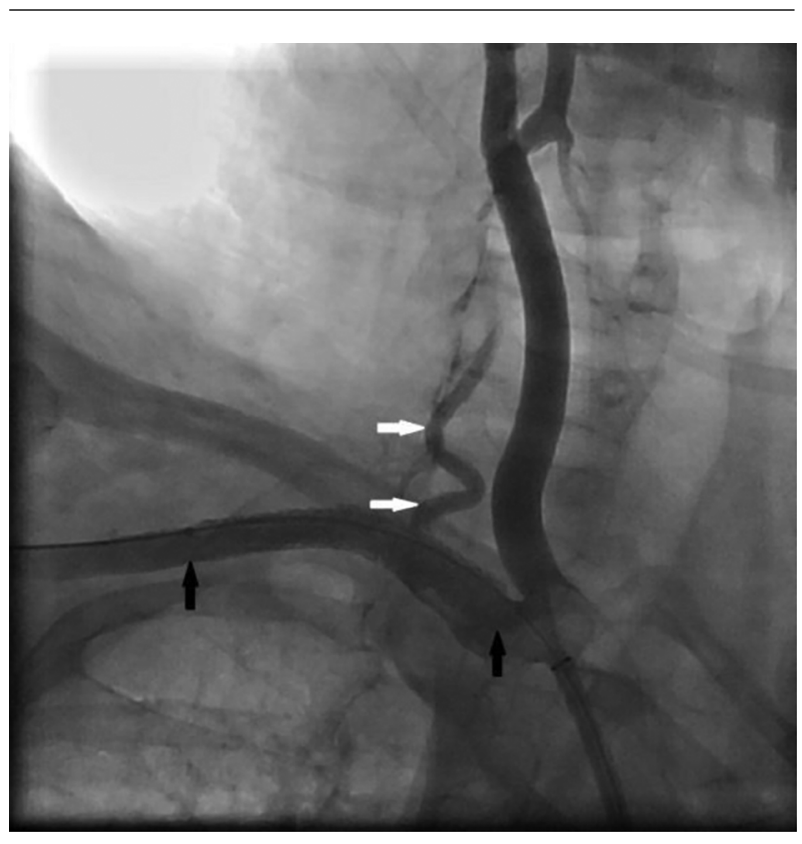

Figure 4. Angiography after successful management shows patent subclavian artery and stent in place (black arrows). There is no extravasation. Right vertebral artery (white arrows) is also noted.

to condition of the patient and also the exact location of the arterial injury (5).

We tried not to only withdraw the catheter and apply external pressure. Evidence suggests that simply removing the misplaced intra-arterial venous catheter without any other intervention is not a safe procedure especially if a large bore catheter was the blame and the subclavian or brachiocephalic arteries were injured; since the defect is too big for external hemostasis and there is no direct route for effective external pressure. This technique has caused more serious complications including massive hemorrhage, airway compromise, hematoma, stroke, and pseudoaneurysm $(2,3,8)$. Based on these data, the patient's age, and history we considered embedding a stent graft over the injured area.

It has been reported previously that using peripheral covered stents can successfully manage serious subclavian artery injuries after iatrogenic puncture $(2,3,5-7)$. Use of a covered stent is especially attractive in cases in which the subclavian artery is dissected, there is a pseudoaneurysm, or there is persistent bleeding from the injury site (5). When choosing a covered stent, the advantage of a balloonexpandable stent is very controlled deployment and flexible sizing, as the stent can be further expanded with the use of larger balloons. In addition, covered stents $5-6 \mathrm{~mm}$ in diameter can be inserted through a 6-F guide sheath and stents as large as $12 \mathrm{~mm}$ in diameter can be inserted through an 8-F sheath (5).

Based on the patients' file notes the vascular surgeon used Seldinger technique to perform a catheterization without fluoroscopic guidance. The primary trocar puncture using 18-gauge needle was ultrasound-guided. No arterial backflow was seen during the first puncture, but there was small resistance for guidewire and dilator advance. The guidewire might have entered the subclavian artery and since no fluoroscopic guidance was used, the dilatators and catheter were introduced and fixed in the artery. Since the patient had several former attempts to catheterize the RIJ vein, we hypothesize previous healed subtle arteriovenous injuries were prone to guidewire entrance, especially if they were introduced by force. Also Oropello et al. have also mentioned such an injury mechanism while placing central catheters (9).

Dilators can perforate a blood vessel or cardiac chamber if they are forced in too deeply, and they may have an even greater propensity for causing perforation than catheters, which are softer. Because the dilator is somewhat stiff, the wire may not be able to successfully guide it through the vein. So, the other hypothesis is that the sheath and dilatator injured the wall of the vessels and entered the artery. As a consequence, the catheter was fixed in the subclavian artery. Dilators are used to dilate the skin and subcutaneous tissue; they should not be pushed further into the vein $(8,9)$.

The preferred insertion site for tunneled cuffed venous dialysis catheters or port catheter systems is the right internal jugular vein. Ultrasound should be used in the placement of catheters (1). Using fluoroscopy to guide guidewire course in a non-emergent situation is a verified clinical procedure which helps to place central lines with less obstacle. In addition, the position of the tip of any central catheter should be verified radiologically (1).

In conclusion, use of ultrasonography and fluoroscopy as a guide for catheterization results in fewer complications especially arterial injuries. When one faces an advertent arterial catheterization, simply removing the catheter may cause an irreversible condition especially if the catheter has a large diameter. The catheter should be left in place. The advent of endovascular management makes it a preferable choice in treating a patient with this condition, especially for arterial injuries below the clavicle and aged patients.

\section{Footnotes}

Authors' Contributions: None declared.

Conflict of Interest: None declared.

Financial Disclosure: None declared. 
Funding/Support: None declared.

\section{References}

1. Vascular Access Work G. Clinical practice guidelines for vascular access. Am J Kidney Dis. 2006;48 Suppl 1:S176-247. doi: 10.1053/j.ajkd.2006.04.029. [PubMed:16813989].

2. Pikwer A, Acosta S, Kolbel T, Malina M, Sonesson B, Akeson J. Management of inadvertent arterial catheterisation associated with central venous access procedures. Eur JVasc Endovasc Surg. 2009;38(6):707-14. doi: 10.1016/j.ejvs.2009.08.009. [PubMed: 19800822].

3. Guilbert MC, Elkouri S, Bracco D, Corriveau MM, Beaudoin N, Dubois MJ, et al. Arterial trauma during central venous catheter insertion: Case series, review and proposed algorithm. J Vasc Surg. 2008;48(4):918-25. discussion 925. doi: 10.1016/j.jvs.2008.04.046. [PubMed: 18703308].

4. Wadhwa R, Toms J, Nanda A, Abreo K, Cuellar H. Angioplasty and stenting of a jugular-carotid fistula resulting from the inadvertent placement of a hemodialysis catheter: case report and review of literature. Semin Dial. 2012;25(4):460-3. doi: 10.1111/j.1525-139X.2011.01005.x. [PubMed: 22151562].
5. Abi-Jaoudeh N, Turba UC, Arslan B, Hagspiel KD, Angle JF, Schenk WG, et al. Management of subclavian arterial injuries following inadvertent arterial puncture during central venous catheter placement. $J$ Vasc Interv Radiol. 2009;20(3):396-402. doi: 10.1016/j.jvir.2008.12.409. [PubMed: 19167239].

6. du Toit DF, Lambrechts AV, Stark H, Warren BL. Long-term results of stent graft treatment of subclavian artery injuries: management of choice for stable patients? J Vasc Surg. 2008;47(4):739-43. doi: 10.1016/j.jvs.2007.11.009. [PubMed: 18242938].

7. Schonholz CJ, Uflacker R, De Gregorio MA, Parodi JC. Stent-graft treatment of trauma to the supra-aortic arteries. A review.J Cardiovasc Surg (Torino). 2007;48(5):537-49. [PubMed: 17989623].

8. Kulvatunyou N, Heard SO, Bankey PE. A subclavian artery injury, secondary to internal jugular vein cannulation, is a predictable rightsided phenomenon. Anesth Analg. 2002;95(3):564-6. table of contents. doi: 10.1213/00000539-200209000-00012. [PubMed: 12198037].

9. Oropello JM, Leibowitz AB, Manasia A, Del Guidice R, Benjamin E. Dilator-associated complications of central vein catheter insertion: possible mechanisms of injury and suggestions for prevention. J Cardiothorac Vasc Anesth. 1996;10(5):634-7. doi: 10.1016/S10530770(96)80142-X. [PubMed: 8841872]. 